\title{
El cine en el Mercosur. El proceso de integración regional y las asimetrías de la industria cinematográfica \\ Marina Moguillansky
}

Mairna Moguillansky es Becaria Doctoral de CONICET en el Instituto de Altos Estudios Sociales (IDAES) de la UNSAM.

Mail: mmoguillansky@fibertel.com.ar

\section{resumen}

Este artículo discute las repercusiones del proceso de integración regional del Mercosur en el sector cinematográfico de los países miembro, a partir del análisis de las políticas nacionales y regionales que se han desarrollado desde su creación. Se describen las asimetrías estructurales y de políticas públicas en el sector cinematográfico entre los países miembro del Mercosur, especialmente marcadas entre los países grandes y los pequeños en todas las dimensiones consideradas. Luego, se analizan las iniciativas de políticas regionales que se han desarrollado en el Mercosur con el objetivo de paliar estas asimetrías en el sector cinematográfico: los acuerdos de co-distribución bilaterales, la creación de la Reunión Especializada de Autoridades Cinematográficas del Mercosur (RECAM) y del Foro de Competitividad para el Sector Cinematográfico.

\section{summary}

This article discusses the consequences of the Mercosur regional integration process on the cinema sector focusing on the analysis of national and regional cinematographic policies. We describe asymmetries in both the industrial structure and public policies between Mercosur members, especially relevant when comparing Argentina and Brazil with Uruguay and Paraguay. Then we analyze recent regional initiatives in cinematographic policies at the Mercosur, designed to reduce asymmetries: bilateral co-distribution agreements, the creation of a Special Reunion of Cinema and Audiovisual Authorities of Mercosur (RECAM), and the Competitiveness Forum for Cinematographic Industries.

\section{palabras clave}

política cinematográfica /

\section{keywords}

asimetrías cinematographic policies / asymmetries / regional integration / Mercosur 


\section{Introducción}

Este artículo discute las repercusiones del proceso de integración regional del Mercosur en el sector cinematográfico de los países miembro ${ }^{1}$, a partir del análisis de las políticas nacionales y regionales que se han desarrollado desde su creación. Este tema cobra una nueva relevancia al ser directamente afectado por la posible modificación de la regulación audiovisual en Argentina, a través del proyecto de Ley de Servicios de Comunicación Audiovisual que está siendo discutido actualmente en la Argentina. Se trata de un sector clave por su creciente importancia económica y por su rol político-cultural, a tal punto que ha sido uno de los puntos de conflicto suscitado en la firma del Tratado de Libre Comercio de las Américas (TLCAN) y en las rondas de negociación del GATS.

Los tratados de integración regional existentes se han dado muy distintas configuraciones legales con respecto a las industrias culturales y el sector audiovisual en particular. La forma en que los bloques regionales lidian con las industrias audiovisuales se vincula con tres factores centrales: a) la estructura industrial de cada país; b) las políticas domésticas sobre el sector; c) las distancias culturales (Galperín, 1999). En este sentido, la Unión Europea, el Tratado de Libre Comercio de América del Norte (TLCAN) y el Mercosur constituirían tres modelos diferentes de enfocar la cuestión de las industrias audiovisuales y la integración. El caso del Mercosur supone un desafío para el análisis puesto que ha existido un vacío casi absoluto de políticas regionales para el sector audiovisual hasta el año 2003. Esta ausencia responde a la matriz típicamente latinoamericana en el área de las políticas culturales, que ha implicado una desatención sistemática hacia los sectores de consumo masivo y las industrias culturales.

Nuestro análisis de las políticas regionales en el sector audiovisual del Mercosur se centra en el problema de las asimetrías intrarregionales y sus expresiones en el mercado y las industrias cinematográficas. En el Mercosur existen asimetrías muy profundas que han dificultado la implementación de las metas de integración que se planteaba el proyecto. Esto se ha visto agravado por el contexto histórico que acompañó la gestación del proyecto del Mercosur, signado por el predominio de las teorías del regionalismo abierto de los años 80 y 90, que pusieron poco énfasis en la corrección de asimetrías, bajo el supuesto de que el gran beneficio del mercado ampliado era mucho mayor para los países pequeños que para los grandes. En este sentido, el Mercosur siguió la línea establecida en la Ronda Uruguay del GATT, que planteaba la ampliación de mercados y la reciprocidad de las negociaciones como aspectos centrales para el desarrollo económico.

El consenso de los años iniciales del regionalismo abierto - que para algunos era más bien neoliberalismo (Briceño Ruiz, 2003: 142)-confiaba en que la ampliación de los mercados sería un gran beneficio para los países pequeños. Esta creencia provocó una ausencia de políticas para afrontar las asimetrías estructurales entre países grandes y pequeños, ya que no se lo consideraba un problema. Dicho diagnóstico fue pronto desmentido por los problemas que encontraron distintos proyectos desarrollados bajo este esquema. Por un lado, las enseñanzas de la Unión Europea y su adopción de políticas de reducción de asimetrías colaboraron 
al proveer el ejemplo de un modelo de integración no limitado a la eliminación de barreras. Por otra parte, la integración iniciada en el Mercosur, la CAN y el NAFTA mostró los límites de la propuesta original.

A partir de la Ronda de Doha se empieza a considerar un modelo de integración más proactivo y vinculado al desarrollo, con prioridad en la reducción de asimetrías entre los países miembro. En consonancia con el cambio de clima de ideas, en los últimos años el Mercosur comenzó a evaluar políticas que atendían a las asimetrías existentes. Teniendo en cuenta las dificultades crecientes que se estaban enfrentando tras las sucesivas devaluaciones de la moneda brasileña desde el año 2000 y la crisis argentina, en la Cumbre de Montevideo del año 2003 se toman las primeras medidas destinadas a evaluar políticas que combatieran estas asimetrías. En los últimos años además comenzaron a hacerse oír cada vez con mayor fuerza los reclamos de los países más pequeños, Uruguay y Paraguay. La ausencia de políticas compensatorias frente a las asimetrías, que se impuso durante la primera década del Mercosur, encontraría un límite en la necesidad de consensuar políticas para contener a estos países, frente a la política del bilateralismo norteamericano que resultaba tentadora para algunos de ellos.

\section{Integración regional y asimetrías}

Las asimetrías pueden en términos generales clasificarse en dos tipos: a) asimetrías estructurales y b) asimetrías de políticas públicas. Las asimetrías estructurales son aquellas que no pueden modificarse a corto plazo, y en algunos casos sencillamente no pueden modificarse sino que a lo sumo se puede proveer un contrapeso: los contrastes geográficos, las diferencias de tamaños en la población, la economía, la riqueza, los mercados, diferencias de infraestructura, de desarrollo industrial sectorial o general, de desarrollo cultural, etc. Las asimetrías de políticas son aquellas generadas por las distancias entre los esquemas de políticas de distintos tipos: ya se trate de políticas fiscales o monetarias, de políticas de incentivos a la producción, subsidios públicos, políticas de financiamiento, etc. Las asimetrías de políticas pueden en muchos casos ser el resultado de asimetrías de recursos, a su vez vinculadas con las asimetrías estructurales. Es importante determinar el tipo de asimetrías que se enfrenta en cada caso, puesto que cada tipo de asimetría requiere una política diferente: para las asimetrías estructurales es necesaria una planificación a largo plazo coordinada a nivel regional, mientras que las asimetrías de política requieren armonización de las políticas nacionales.

\section{Las asimetrías estructurales en el sector cinematográfico del Mercosur}

Los países del Mercosur, como se ha dicho, presentan marcadas asimetrías estructurales que clasificaremos en las que se refieren al tamaño de los mercados cinematográficos y las que atañen al desarrollo de la industria cinematográfica. Como ha sido establecido en la bibliografía, el desarrollo de la industria se ve condicionado por el tamaño del mercado interno. 


\section{a) Tamaño de los mercados}

Los países miembro del Mercosur tienen mercados potenciales para el consumo de cine marcadamente asimétricos. Podemos en principio distinguir entre los dos países grandes (Brasil y Argentina) y los dos pequeños (Paraguay y Uruguay), pero además debemos tener en cuenta que entre los países de mayores mercados también existe una diferencia significativa por el mayor tamaño del mercado brasileño. Para la consideración del mercado cinematográfico en lo que a espectadores respecta debemos tener en cuenta dos cuestiones: el tamaño de la población y su disposición al consumo de cine. Una mayor población no se traduce automáticamente en un mayor consumo de cine: existen mediaciones vinculadas con la distribución del ingreso y con tradiciones culturales.

\section{Tabla $\mathbf{N}^{0} 1$}

Principales indicadores de tamaño del mercado potencial cinematográfico en los países miembro del Mercosur

\begin{tabular}{|c|c|c|c|c|}
\hline & $\begin{array}{c}\text { Superficie } \\
\left(\mathbf{k m}^{2}\right)\end{array}$ & $\begin{array}{c}\text { Población } \\
(\text { miles })\end{array}$ & $\begin{array}{c}\text { PIB } \\
\text { corriente } \\
\mathbf{2 0 0 6} \\
\text { (millones } \\
\text { USS) }\end{array}$ & $\begin{array}{c}\text { PIB per } \\
\text { cápita } \\
\text { (USS) }\end{array}$ \\
\hline Argentina & 3.761 .274 & 39.356 & 211.669 & 5.378 \\
\hline Brasil & 8.514 .215 & 183.988 & 1.067 .706 & 5.803 \\
\hline Paraguay & 406.752 & 6.216 & 8.773 & 1.411 \\
\hline Uruguay & 176.215 & 3.455 & 19.221 & 3.836 \\
\hline Total & 12.858 .456 & 235.860 & 1.307 .369 & 4103 \\
\hline
\end{tabular}

Fuente: INDEC, IBGE, Büttner (2007)

Las asimetrías entre los países del Mercosur son importantes en todos los indicadores considerados: territorio, población, producto bruto interno y producto bruto interno per cápita. La población es uno de los factores clave para evaluar el tamaño potencial del mercado cinematográfico y, en este ítem, Brasil tiene una población que supera en casi cinco veces a la argentina. Del mismo modo, la población de Argentina sextuplica a la de Paraguay. Este último país casi duplica a la población de Uruguay, pero como veremos en el siguiente apartado, la diferencia entre los países pequeños se ve muy matizada porque la capacidad adquisitiva promedio de la población uruguaya y su cultura cinematográfica se combinan para producir un consumo de cine por habitante muy alto.

Las asimetrías entre los miembros del Mercosur son también muy marcadas en lo que respecta al Producto Bruto Interno, donde nuevamente Brasil quintuplica el valor de Argentina, y el PBI argentino es diez veces el de Uruguay, que a su vez duplica el PBI paraguayo. Estas diferencias son centrales puesto que determinan 
diferentes capacidades de inversión en políticas públicas de apoyo al sector cinematográfico.

Las asimetrías estructurales que estamos considerando se manifiestan en fuertes diferencias en los tamaños del público potencial para el cine, como veremos en la siguiente tabla.

\section{Tabla $\mathbf{N}^{0} 2$}

Indicadores del tamaño del mercado cinematográfico en los países miembro del Mercosur en el año 2005

\begin{tabular}{|c|c|c|c|c|}
\hline & $\begin{array}{c}\text { Espectadores } \\
\text { anuales } \\
\text { promedio en } \\
\text { millones }\end{array}$ & $\begin{array}{c}\text { Recaudación } \\
\text { bruta en salas } \\
\text { (miles de } \\
\text { USS) }\end{array}$ & $\begin{array}{c}\text { Cantidad de } \\
\text { salas }\end{array}$ & $\begin{array}{c}\text { Índice de } \\
\text { concurrencia } \\
\text { persona/año }\end{array}$ \\
\hline Argentina & 36,6 & 80.345 & 999 & 0,97 \\
\hline Brasil & 99,1 & 248.192 & 1.982 & 0,61 \\
\hline Paraguay & 0,6 & $\mathrm{~s} / \mathrm{d}$ & 57 & 0,10 \\
\hline Uruguay & 2,5 & 7.300 & 83 & 0,66 \\
\hline Total & $\mathbf{1 3 8 , 8}$ & $\mathbf{3 3 5 . 8 3 7}$ & $\mathbf{3 1 2 1}$ & $\mathbf{0 , 5 9}$ \\
\hline
\end{tabular}

Fuente: OMA (2007)

Las asimetrías son muy importantes considerando el tamaño del mercado cinematográfico de los distintos países del Mercosur. Brasil cuenta con el 71\% de los espectadores de la región, con los cuales obtiene el $74 \%$ de la recaudación en salas de cine, a pesar de que el índice de concurrencia por persona no es muy alto $(0,61)$. Además, Brasil posee el $63 \%$ de las salas de la región. A mucha distancia se ubica el mercado cinematográfico argentino, que presenta el índice más alto de concurrencia $(0,97$ entradas por persona por año), y cuenta con el $26 \%$ de los espectadores de la región. Esto quiere decir que entre Brasil y Argentina acaparan el 97\% del mercado cinematográfico del Mercosur en términos de espectadores. Las pantallas de Uruguay, aún cuando este país presenta un alto índice de concurrencia y una importante cantidad de salas en relación con el tamaño de su población, sólo representan el $2 \%$ de la recaudación del mercado cinematográfico de la región.

\section{b) Desarrollo de la industria cinematográfica}

El desarrollo de la industria cinematográfica se ve fuertemente condicionado al tamaño del mercado potencial para sus productos, como ha sido demostrado en general por la bibliografía que estudia el desempeño de las industrias culturales. Como se trata de bienes cuyo costo inicial es alto y sus costos marginales tienden a cero, requieren grandes economías de escala para resultar rentables. A continuación, veremos un cuadro con indicadores del tamaño respectivo de 
las industrias cinematográficas de los países del Mercosur, donde se comprueba que las asimetrías de los mercados son correlativas con las de desarrollo de las industrias del cine.

\section{Tabla $\mathbf{N}^{\mathbf{0} 3}$}

Indicadores del tamaño de la industria cinematográfica en los países miembro del Mercosur

\begin{tabular}{|l|c|c|c|c|}
\hline & $\begin{array}{c}\text { Producción } \\
\text { anual de } \\
\text { películas } \\
(2005)\end{array}$ & $\begin{array}{c}\text { Estrenos } \\
\text { anuales } \\
(2005)\end{array}$ & $\begin{array}{c}\text { Estrenos de } \\
\text { películas } \\
\text { nacionales } \\
(2005)\end{array}$ & $\begin{array}{c}\text { Cantidad } \\
\text { de empleos } \\
\text { en el sector } \\
(2000)\end{array}$ \\
\hline Argentina & 41 & 245 & 65 & 36.000 \\
\hline Brasil & 51 & 278 & 40 & 20.143 \\
\hline Paraguay & - & 90 & 1 & 2 \\
\hline Uruguay & 5 & 166 & 5 & 200 \\
\hline Total & $\mathbf{9 7}$ & $\mathbf{7 9 9}$ & $\mathbf{1 1 1}$ & $\mathbf{5 6 . 3 4 3}$ \\
\hline
\end{tabular}

Fuente: Elaboración propia en base a datos de Getino (2007), INCAA, SICA, Filmes B, Mastrini y Becerra (2006) y sitio cinesparaguay

Si prestamos atención a la producción anual de películas en los países del Mercosur, podemos observar que las asimetrías son tan importantes que se cuenta representantes en tres de las cuatro categorías establecidas por la UNESCO: dos países de producción mediana (de 20 a 199 películas), un país de producción escasa ( 1 a 19 películas) y un país sin producción. Las asimetrías en la producción cinematográfica guardan estrecha relación con las asimetrías en los tamaños del mercado, descritas en el apartado anterior.

\section{Asimetrías de políticas públicas en la cinematografía del Mercosur}

Las asimetrías en políticas públicas referidas al sector cinematográfico en los países del Mercosur son también muy importantes y se vinculan con la existencia de tradiciones de producción y con el desarrollo del sector en cada país. Argentina cuenta con una larga historia de regímenes de protección a la industria cinematográfica, si bien se han alternado con períodos de apertura y liberalización de las regulaciones. Asimismo, en el año 1993 se sancionó la Ley del Cine ( $\left.\mathrm{N}^{\circ} 24.377\right)$ que es considerada una de las más avanzadas de América Latina, por cuanto instaló un mecanismo de financiamiento cruzado entre el cine y la televisión. Brasil también ha tenido una importante tradición de intervención estatal en la industria aunque menos acompañada por la formalización legal. Sin embargo, a partir de la Ley Rouanet y la Ley del Audiovisual, este país también cuenta con una legislación específica para el sector que establece mecanismos de subsidio a la producción. 
Paraguay y Uruguay, en cambio, no cuentan con leyes específicas del sector. En el caso de Paraguay, el Ministerio de Cultura acaba de crearse y se encuentra todavía en una fase de inicio de sus actividades. El Uruguay sí cuenta con un Ministerio de Educación y Cultura y está discutiendo actualmente la aprobación de una ley de cine, pero hasta la fecha no posee legislación específica para el sector.

\section{a) Políticas de financiamiento a la producción cinematográfica}

Desde los inicios de la década de 1990, Argentina y Brasil renovaron sus legislaciones sobre el sector cinematográfico impulsando líneas de financiamiento para la producción de películas. A través de vías diferenciadas pero con resultados bastante similares, ambos países cedieron recursos públicos para subsidiar u otorgar créditos a la producción. En el caso de Brasil se trata de una legislación de fomento al mecenazgo que permite a las empresas descontar parte de los pagos de impuestos a través de la inversión en proyectos culturales. En Argentina, la Ley de Cine fijó impuestos que constituyeron el Fondo Cinematográfico con el cual se otorgan subsidios y créditos blandos para la producción cinematográfica. Entre los subsidios se distinguen los de "recuperación industrial" y los de "medios electrónicos". La diferencia con respecto al mecanismo brasileño, es que en el caso argentino el Estado se reserva la potestad de seleccionar los proyectos en los que se invierten los fondos públicos, mientras que en Brasil son las propias empresas las que toman estas decisiones. En el siguiente cuadro resumimos las diferentes modalidades empleadas en los países miembro del Mercosur para financiar la producción cinematográfica a través de fondos públicos.

Tabla $\mathbf{N}^{0} 4$

Subsidios y créditos a la producción cinematográfica en el año 1996-2006

\begin{tabular}{|c|c|c|c|c|}
\hline & Subsidios & $\begin{array}{c}\text { Incentivo } \\
\text { fiscal }\end{array}$ & Créditos & Premios \\
\hline Argentina & $\mathrm{X}$ & - & $\mathrm{X}$ & $\mathrm{X}$ \\
\hline Brasil & - & $\mathrm{X}$ & - & $\mathrm{X}$ \\
\hline Paraguay & - & - & - & - \\
\hline Uruguay & - & $\mathrm{X}$ & - & $\mathrm{X}$ \\
\hline
\end{tabular}

Fuente: Elaboración propia en base a datos de ANCINE, INCAA y FONA

Argentina tiene mecanismos de otorgamiento de subsidios y créditos blandos, y cuenta también con concursos que brindan premios de diversa índole a los proyectos ganadores. En los últimos años se ha estudiado la posibilidad de implementar una ley de mecenazgo pero no se ha concretado por el momento. Brasil utiliza el incentivo fiscal como su principal mecanismo de financiamiento, permitiendo a las empresas descontar impuestos mediante la inversión en proyectos culturales. Las principales protagonistas de esta política han sido empresas estatales como Petrobrás y el Banco do Brasil. Además, este país cuenta con premios otorgados a 
través de concursos de proyectos. No se ha implementado hasta el momento una política de subsidios ni créditos especiales para el sector.

En cuanto a los países más pequeños, la situación más crítica es la de Paraguay, que no cuenta con ninguna línea de financiamiento significativa para la producción de películas. Según Francisco Pérez-Maricevich (2003: 450) "en Paraguay no existen fuentes estatales ni privadas que inviertan en el audiovisual debido a que los costos son muy altos y muy difícil la recuperación de la inversión". Debemos destacar, sin embargo, que actualmente se está promoviendo la formulación de una ley de cine para fomentar el desarrollo de la industria. Uruguay, por su parte, cuenta desde el año 1995 con un premio para la producción de ficción y documentales que ha dinamizado considerablemente su cinematografía. Además, en 1996 se estableció un régimen por el decreto 338/996 que permite al Poder Ejecutivo brindar incentivos fiscales a las empresas que contribuyan con proyectos culturales. En mayo de 2008, este país aprobó una nueva ley de cine que creará mayores fondos para el financiamiento de la producción cinematográfica.

\section{Tabla $\mathbf{N}^{0} 5$}

Financiamiento público para la producción cinematográfica 1995-2005 (en miles de dólares)

\begin{tabular}{|c|c|c|c|c|c|}
\hline & Brasil & Argentina & Paraguay & Uruguay & Total \\
\hline $\mathbf{1 9 9 5}$ & 26674 & $\mathrm{~s} / \mathrm{d}$ & 0 & 10 & 26.684 \\
\hline $\mathbf{1 9 9 6}$ & 70.752 & 35.208 & 0 & 60 & 106.020 \\
\hline $\mathbf{1 9 9 7}$ & 95.812 & $\mathrm{~s} / \mathrm{d}$ & 0 & 240 & 96.052 \\
\hline $\mathbf{1 9 9 8}$ & 55.978 & $\mathrm{~s} / \mathrm{d}$ & 0 & 240 & 56.218 \\
\hline $\mathbf{1 9 9 9}$ & 27.276 & 50.906 & 0 & 180 & 78.362 \\
\hline $\mathbf{2 0 0 0}$ & 26.446 & 49.407 & 0 & 180 & 76.033 \\
\hline $\mathbf{2 0 0 1}$ & 36.255 & 45.756 & 0 & 180 & 82.191 \\
\hline $\mathbf{2 0 0 2}$ & 23.820 & 11.722 & 0 & 180 & 35.722 \\
\hline $\mathbf{2 0 0 3}$ & 40.338 & 17.289 & 0 & 0 & 57.627 \\
\hline $\mathbf{2 0 0 4}$ & 54.317 & 19.015 & 0 & 160 & 73.492 \\
\hline $\mathbf{2 0 0 5}$ & 58.593 & 21.282 & 0 & 174 & 80.049 \\
\hline Total & 516.261 & 250.585 & 0 & 1.604 & 768.450 \\
\hline
\end{tabular}

Fuente: Elaboración propia en base a datos de ANCINE, FONA y AFIP

Atendiendo a la distribución regional del dinero destinado al sector cinematográfico, nuevamente podemos constatar que existen grandes asimetrías en las políticas de financiamiento de la producción, consecuentes con las capacidades diferenciales en función del tamaño de los mercados, el desarrollo de las econo- 
mías y el de la industria cinematográfica en particular. En principio, Brasil duplica la inversión que realiza Argentina en financiamiento de la producción. Pero las asimetrías son mayores si contrastamos la situación de los dos países mayores con la de los menores: aquí requeriríamos verdaderamente de un concepto más radical; tan grande es la diferencia entre Brasil y Argentina, que juntos explican el 99,8\% de la inversión pública en financiamiento de la producción cinematográfica de la región, y los dos pequeños, Paraguay y Uruguay, que cuentan sólo con el 0,2\% restante. En el caso de Paraguay, como ya mencionamos, no hay financiamiento alguno; en el Uruguay, hay una línea de premios que, si bien es modesta, ha tenido cierto impacto en la industria de ese país. Ambos países recurren a estrategias de coproducción y a fondos internacionales para poder producir alguna obra cinematográfica.

En efecto, los países más chicos del Mercosur se ven en una situación muy asimétrica ya que prácticamente no cuentan con financiamiento para la producción cinematográfica. Aún así, se puede diferenciar a Uruguay por algunos avances que han realizado en la dirección de establecer apoyo estatal a la producción. En este país se creó en el año 1994 el Instituto Nacional Audiovisual (INA), dependiente del Ministerio de Educación y Cultura pero no cuenta con un presupuesto que le permita establecer subsidios o créditos para la cinematografía. Uruguay además participa del Programa Ibermedia pero ha tenido dificultades en afrontar el pago de la cuota, por lo que estuvo cerca de ser sancionado con la suspensión de sus proyectos. Desde el año $1996^{2}$, en Uruguay se comenzó a implementar un premio para la realización de películas que, a pesar de ser bastante limitado en su magnitud, ha permitido dinamizar la producción. Se trata del Fondo para el Fomento y Desarrollo de la Producción Audiovisual Nacional (FONA) creado por la Intendencia Municipal de Montevideo; el mismo canaliza aportes privados, de la comuna y del Ministerio de Educación y Cultura. Por último, el parlamento uruguayo está pronto a aprobar la Ley de Cine y Audiovisual ${ }^{3}$ que creará el Instituto de Cine y Audiovisual de Uruguay; establecerá un fondo anual de un millón de dólares para fomentar la producción

\section{b) Cuota de pantalla}

La situación de los países miembro del Mercosur en cuanto a las políticas de establecimiento de cuotas preferenciales para la producción nacional en sus pantallas cinematográficas es muy asimétrica, reflejando las respectivas capacidades de producción de películas. Como vemos en el siguiente cuadro, Brasil y Argentina han implementado mecanismos para promover los estrenos y la permanencia en salas de sus películas, mientras que Paraguay y Uruguay no tienen políticas en ese sentido.

Cabe señalar que Paraguay y Uruguay no poseen cuotas de pantalla ni sería esperable que las establecieran en tanto no cuenten con la suficiente producción de cine nacional para proveer a las pantallas.

Brasil tiene desde hace años cuota de pantalla para proteger su producción. Desde la creación de ANCINE en el año 2003, se reformuló el sistema de cuota de 
pantalla para el cine nacional a través de resoluciones anuales que fijan el número de títulos y la cantidad de días que deberá estar cartel, en cantidades graduadas según la cantidad de salas que posea el establecimiento. El mínimo es de 28 días anuales y dos títulos brasileños por sala, luego estas cantidades aumentan progresivamente. Cada año se va estableciendo por decreto de ANCINE el mínimo de días y la cantidad de títulos que deben estrenarse, según estudios técnicos que realiza esta agencia estatal.

\section{Tabla $\mathbf{N}^{0} 6$}

Mecanismos de protección de la producción cinematográfica nacional en los países del Mercosur (2007)

\begin{tabular}{|c|c|c|c|}
\hline & $\begin{array}{c}\text { Cuota de } \\
\text { pantalla }\end{array}$ & $\begin{array}{c}\text { Media de } \\
\text { continuidad }\end{array}$ & Días \\
\hline Argentina & $\mathrm{X}$ & $\mathrm{X}$ & \\
\hline Brasil & $\mathrm{X}$ & & $\mathrm{X}$ \\
\hline Paraguay & - & - & - \\
\hline Uruguay & - & - & - \\
\hline
\end{tabular}

Fuente: síntesis del cuadro elaborado por Getino (2007)

Argentina cuenta con cuota de pantalla desde el año 2004, fecha en que se reglamentaron la cuota de pantalla y la media de continuidad ${ }^{4}$ previstas en la Ley del Cine (17.741/95). La cuota de pantalla establece que cada sala de cine estrene una película argentina por trimestre. La media de continuidad indica un mínimo porcentaje de la sala por encima del cual la película no puede ser retirada de cartel. Pero en poco tiempo se tornó evidente que los exhibidores no estaban cumpliendo con estas exigencias y que las medidas les dejaban mucho margen de acción. Ello dio lugar a una nueva política de intervención en la exhibición por parte del INCAA $^{5}$, que con la gestión de Jorge Álvarez aprobó una resolución que introduce varias novedades: 1) la cuota de pantalla se ajusta contemplando distintos tipos de películas según la cantidad de copias con las que se estrenan; 2) se crea un circuito alternativo de exhibición por el cual el INCAA alquila determinadas salas y garantiza la permanencia por dos semanas para las películas que se estrenan con menos copias; 3) establece un calendario de estrenos de películas nacionales; y 4) crea una "Comisión de Aplicación y Autorregulación de la cuota de pantalla" con representantes de los productores cinematográficos. Estas iniciativas han generado mucho debate en el sector y airadas protestas de los exhibidores multipantalla, a pesar de que algunos analistas señalan que: "se trata del sistema más benévolo para el exhibidor como forma de regulación. En otros países se los obliga a ocupar una cantidad de días al año con cine nacional funcione éste o no. Aquí, en el caso de no alcanzar la película la media de la sala, la misma baja de cartel" (Arias, 2005). 


\section{Consecuencias del escenario asimétrico en el sector audiovisual}

El resultado de las asimetrías estructurales y de políticas que hemos descrito en los sectores audiovisuales de los países del Mercosur genera una situación poco propicia para la integración y la cooperación. El subdesarrollo de los mercados en Paraguay y Uruguay desincentiva las estrategias de circulación de obras audiovisuales en estos países, así como el desarrollo autónomo de producción cinematográfica. A su vez, debemos tener en cuenta que, como demuestran distintos estudios sobre cultura audiovisual, el consumo de imágenes propias es más atractivo para la población, tiene efectos positivos en términos identitarios y además mejora en general la performance de la industria cinematográfica. En ese sentido, la carencia de cinematografías propias desperdicia la potencial ventaja doméstica y limita el interés de los espectadores por el cine, reforzando la situación de inactividad.

Por otra parte, como veremos en el siguiente apartado, las enormes asimetrías existentes entre Brasil y Argentina por un lado, y Uruguay y Paraguay por el otro, tornan difícil la implementación de políticas de coordinación e integración cinematográficas como la coproducción o codistribución, puesto que requieren de una contrapartida en muchos casos inexistente, o bien la cuota de pantalla regional que despertaría muchas resistencias en los países pequeños.

La ampliación del mercado potencial para las cinematografías de los países del Mercosur es un beneficio muy importante que podría permitir economías de escala para mejorar la rentabilidad de la actividad industrial. Sin embargo, como ha quedado demostrado en la experiencia de este proceso de integración, ello no es suficiente. Las asimetrías de la región se suman a la configuración del mercado cinematográfico a nivel internacional, en el cual la hegemonía de las majors norteamericanas y sus estrategias de integración y concentración sin límites han generado barreras de entrada muy difíciles de sortear (Moguillansky, 2007).

\section{Algunas políticas implementadas en el Mercosur para corregir las asimetrías}

Desde el año 2003, los países menores del Mercosur comenzaron a plantear quejas por las asimetrías existentes en la región y por la escasísima atención que se les venía prestando en términos de políticas. Una de las principales respuestas que obtuvieron fue la creación del Fondo de Convergencia Estructural del Mercosur (FOCEM). Según los analistas, la aplicación de políticas de discriminación negociada y fondos regionales es muy problemática en el Mercosur porque este bloque reúne a "países de bajos ingresos, con problemas distributivos internos, porque se otorga poca relevancia a las consideraciones de cohesión, y porque existen fuertes resistencias a delegar competencias estatales" (Bouzas, 2005).

En el caso del sector cinematográfico, no se han diseñado políticas específicas para solucionar los problemas generados por las asimetrías entre los países de la región. En realidad, los avances en políticas culturales regionales han sido bastante modestos, aunque se crearon instituciones específicas para el sector cinematográfico y se diseñaron numerosos proyectos. 


\section{La Reunión Especializada de Autoridades Cinematográficas (RECAM)}

La primera decisión intergubernamental para la creación de la RECAM fue adoptada por el Grupo Mercado Común en Montevideo, en diciembre de 2003, considerando "la conveniencia de establecer un foro destinado al análisis y desarrollo de mecanismos de promoción e intercambio de la producción y distribución de los bienes, servicios y personal técnico y artístico relacionados con la industria cinematográfica y audiovisual en el ámbito del MERCOSUR” (Getino, 2006).

En el marco de la RECAM se desarrollaron diversas iniciativas: se emprendió un estudio de los obstáculos a la circulación de obras cinematográficas intra-Mercosur; se promovió y/o apoyó la generación de leyes del cine en Paraguay y Uruguay; se fomentó la realización de festivales de cine del Mercosur; se fomentaron acuerdos de co-distribución, como el que firmaron el Brasil y la Argentina en el año 2004; y se promovió la creación de un Foro de Competitividad para el sector cinematográfico.

Esta última iniciativa, el Foro de Competitividad del Cine, es aún muy reciente para ser evaluada, pero ha despertado entusiasmo en los actores interesados. Los Foros se crean en el ámbito del Programa de Foros de Competitividad de las Cadenas Productivas del MERCOSUR creado por el Consejo Mercado Común (CMC), para aprovechar las ventajas comparativas de los países miembros y para mejorar la competitividad global. En el año 2007 se creó el Foro de Competitividad del Sector Cinematográfico y en el año 2008 tuvo su primera reunión, de la que no han trascendido resultados.

\section{Conclusiones}

Hemos comprobado que entre los países del Mercosur existen fuertes asimetrías estructurales que parten del tamaño geográfico y del desarrollo económico mucho mayor de Brasil y Argentina con respecto a Uruguay y Paraguay. Estas diferencias de base se expresan en las grandes diferencias que se constatan también en cuanto a los mercados cinematográficos y en el desarrollo de las industrias audiovisuales, y además en la capacidad para implementar políticas públicas para el fomento de la actividad en la financiación de la producción cinematográfica, en la estructura legislativa y en la existencia de cuotas de pantalla. Las posibilidades de los Estados para financiar la producción cinematográfica son una expresión muy visible de las asimetrías internas de la región.

De esta manera, las asimetrías existentes se conjugan generando un escenario de escasa interacción entre los respectivos mercados cinematográficos (y unilateralidad de las exportaciones de los grandes hacia los países pequeños) desincentivando las iniciativas de integración y desperdiciando las potencialidades del mercado regional. Esta situación confluye con la configuración del sector cinematográfico a nivel mundial, con fuertes barreras de entrada y que impone la necesidad de economías de escala y de altas inversiones en la producción, tornan estratégica y necesaria la unificación de políticas cinematográficas en el Mercosur. Las iniciativas desarrolladas hasta el momento en el Mercosur han sido insuficientes y poco efectivas, en parte debido a que no han logrado atender a las asimetrías como obstáculos para la integración. 


\section{Referencias}

1. En este trabajo nos limitaremos a considerar como países miembro a Argentina, Brasil, Paraguay y Uruguay. Debido a lo reciente de la incorporación de Venezuela al Mercosur (que todavía no ha sido aprobada por el Parlamento brasileño) no hemos analizado su situación, así como tampoco hemos incluido a Bolivia y Chile, países asociados.

2. El FONA se creó en 1995 y el primer concurso se realizó en el año 1996.

3. A la fecha de redacción de este trabajo, el proyecto fue aprobado por la Cámara de Diputados y aguarda tratamiento en la Cámara de Senadores.

4. A través de la Resolución 2.016, del 28 de junio de 2004.

5. Esta nueva política de regulación entró en vigencia el 1 de septiembre de 2006.

\section{Bibliografía}

R. BOUZAS (2005), "MERCOSUR: Regional governance, asimetrías e integración profunda” en Foro INTAL.

J. BRICEÑO RUIZ (2003), Las teorías de la integración regional, Mérida, Universidad de Los Andes.

J. BÜTTNER (2007), "Instrumentos de combate a las asimetrías regionales: el Fondo para la Convergencia Estructural (FOCEM)", presentado en el XIV Fórum Brasil-Europa.

H. GALPERÍN (1999), Cultural Industries Policy in Regional Trade Agreements: The Case of NAFTA, the European Union and MERCOSUR, Media, Culture, \& Society 21.

O. GETINO (2006), "Negociación e integración en el sector cinematográfico y audiovisual en los países del Mercosur. Antecedentes y experiencias" en www.recam.org

G. MASTRINI y M. BECERRA (2006), Periodistas y magnates. Estructura y concentración de las industrias culturales en América Latina. Buenos Aires, Prometeo.

M. MOGUILLANSKY (2007), “¿Un cine global? Las transformaciones recientes en el mercado cinematográfico" en VII Jornadas de Sociología de la UBA, noviembre, Buenos Aires.

OMA (2007), Aproximación al mercado cinematográfico del Mercosur. Período 2002-2005, en www.oma.recam.org

F. PÉREZ-MARICEVICH (2003), "Informe sobre la situación de las industrias culturales en el Paraguay" en Indústrias Culturais no Mercosur, Brasilia, IBRI.

RECAM (2004), Industria audiovisual uruguaya, en www.recam.org

T. SCHEMBORI y J.C. MANEGLIA (2003), "La industria audiovisual en Paraguay" en www.recam.org

UNESCO (2001), Encuesta sobre cinematografias nacionales, en http://www.unesco.org/culture/ industries/cinema/html

Recibido: 27/03/09. Aceptado: 10/06/09. 